\title{
A Photonics Enabled Millimetre Wave Frequency Domain Spectrometer for Glucose Concentration Sensing
}

\author{
J.P. Seddon, K. Balakier, X. Lin, C. Graham, A.J. Seeds, C.C. Renaud \\ University College London, Department of Electronic \& Electrical Engineering, Torrington Place, London, \\ WC1E 7JE, United Kingdom
}

\begin{abstract}
This study reports the experimental demonstration of a free space frequency domain self-heterodyne spectrometer utilising a high-speed Uni-Travelling Carrier photodiode (UTCPD) as a coherent receiver. The transmission signal through the glucose solution was measured in the $\mathrm{V}$-band $(50-75 \mathrm{GHz})$ frequency range with a frequency resolution step of $40 \mathrm{MHz}$. Variations in glucose concentrations of as small as $1 \%$ weight were resolved.
\end{abstract}

\section{INTRODUCTION}

A broadly tuneable frequency domain spectrometer with $\mathrm{MHz}$-range resolution can be developed using photonic components, allowing for extensive frequency agility and small size of the system. The proposed approach exploits the process of heterodyning two optical tones in a photodiode to generate mm-wave signals. A coplanar waveguide integrated UTC-PD is used as an Optically Pumped Mixer (OPM), for heterodyne detection of millimetre wave signals in the $\mathrm{V}$-band as a means to evaluate varying concentrations of glucose in deionized water solutions.

Transmission measurements were made for each sample using 49-75 GHz horn antennas, to create a free space path of $13.5 \mathrm{~cm}$ using the experimental configuration shown in Fig.1. In this arrangement laser 1 and laser 2 are offset in frequency, the offset between the two laser tones is combined using a 50:50 fibre coupler and coupled to a photodiode via optical fibre. The resulting beat note at the offset frequency modulates photoexcited carriers in the photodiode producing an electronic at the beat or heterodyne frequency.

The receiver also utilizes two optical tones also from laser 1 and 2 however in this case the tone from laser 2 is upshifted in frequency by $80 \mathrm{MHz}$ using an Acousto Optic Modulator (AOM). These two tones are then mixed in the UTC PD after amplification using an Erbium Doped Fibre Amplifier (EDFA), amplification is required to reach the saturation point of the photodiode.

High optical pump powers accumulate space charges in the depletion layer of the device, which results in charge storage in the absorber layer[1]. The resulting modulated carrier transit time is thought to be responsible for frequency mixing in the device[2]. When the photonic Local Oscillator is mixed with an offset incoming signal. An Intermediate Frequency (IF) is generated at the offset frequency with some conversion loss. The incoming signal is amplified after the horn antenna using a 50-65 GHz Low Noise Amplifier (LNA) and then injected into the photodiode via a two-resistor power splitter and an Air CoPlanar (ACP) probe. The IF is extracted from the device via the APC probe and the power splitter. Optical isolators were used at the point where the first two 50:50 fibre couples cross to avoid the upshifted signal leaking into the unshifted side as connecting the couplers in this formation allows several back- reflected signals to travel back through the loop and get amplified to create an unwanted $80 \mathrm{MHz}$ beat signal.

The IF power from the OPM was recorded across a band of 49.9 - $67 \mathrm{GHz}$ limited by the cut-off frequency of the electronic equipment (ACP probe and LNA). The sample holder was a 7 $\mathrm{x} 4 \times 1.3 \mathrm{~cm}$ tank made from $3 \mathrm{~mm}$ acrylic, fixed in position between two antennas. Samples were added via pipette and the tank was rinsed with deionized water between samples. Measurements were taken on a small change in percentage weight concentration of $0.05 \%$ weight $(50 \mathrm{mg} / \mathrm{dL})$ and a large change of $5 \%$ weight $(5000 \mathrm{mg} / \mathrm{dL})$. The concentration sizes were in line with samples used in other studies[3] that have clinical relevance for the diagnosis of diabetes.

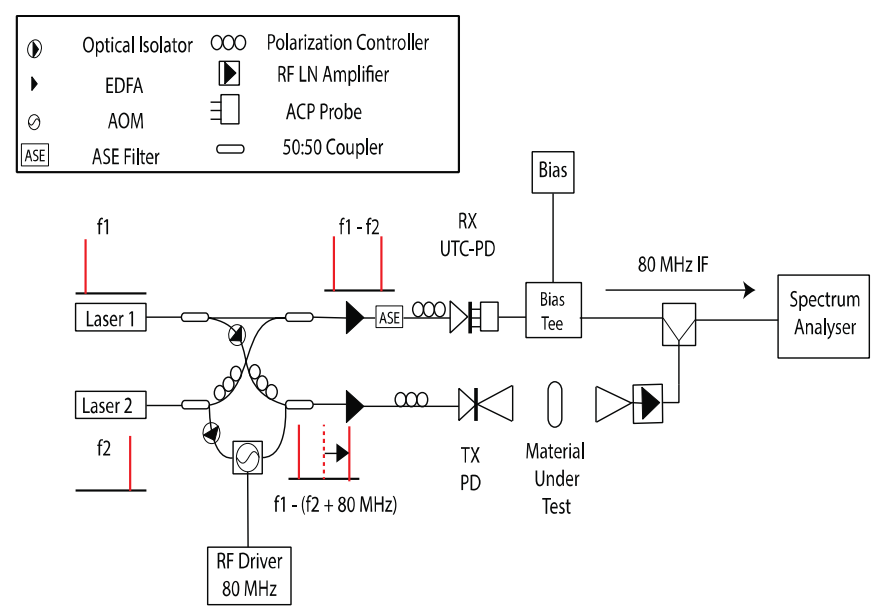

Fig. 1. Schematic diagram of experimental arrangement, the down-converted IF is recorded on a spectrum analyser with $50 \mathrm{KHz}$ resolution bandwidth and $100 \mathrm{KHz}$ video bandwidth. The recorded IF power is an average of 20 traces using the instruments averaging function.

\section{RESUlts}

The effect of concentration variation from $0.05 \%$ to $5 \%$ weight glucose in water on the transmitted mm-wave signal power was recorded along with the DC photocurrent generated by the UTC-PD and is shown in Fig.2 Each frequency point recorded is the average of 20 sweeps from the marker averaging function of the spectrum analyser. The photocurrent was measured as a means of assessing the system stability and if required could provide a method to normalize any drift in the amplitude of the signal, for instance, due to potential optical fibre misalignment over the duration of the measurement. Small changes to the transmitted power are observed for glucose concentration changes of $0.5 \%$, which are in line with previous studies[3], [4]. 


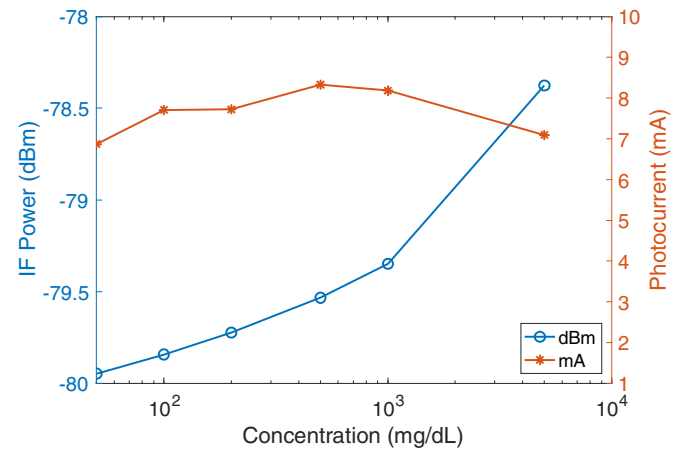

Fig. 2. Change in IF power for a frequency of $52 \mathrm{GHz}$ for increasing concentrations of glucose, DC photocurrent is shown for each measurement showing fluctuations of optical power coupled into the photodiode.

Fig. 3 Shows a second measurement at $65 \mathrm{GHz}$ shows a similar increase in transmission, with an almost equivalent change in transmitted power between 0 and $5000 \mathrm{mg} / \mathrm{dL}$ of $\sim 1.5 \mathrm{~dB}$. Again, a +/- $1 \mathrm{~mA}$ fluctuation in the photocurrent is observed. The photocurrent stability could be improved through full or partial optoelectronic packaging of the receiver photodiode. Stable assembly of the lensed fibre to a device sub-mount should alleviate the photocurrent fluctuations absorbed which are primarily attributed to mechanical or acoustic vibrations.

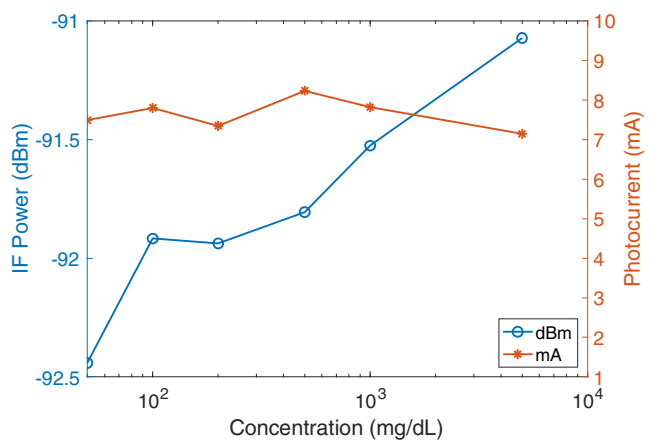

Fig. 3. Change in IF power for a frequency of $65 \mathrm{GHz}$ for increasing concentrations of glucose, DC photocurrent is shown for each measurement showing fluctuations of optical power coupled into the photodiode.

The sensitivity of the receiver photodiode was measured at -30 $\mathrm{dBm}$ across the band for a $10 \mathrm{~dB}$ signal to noise ratio. The main limitation in terms of noise comes from Amplified Spontaneous Emission noise from the photonically driven Local Oscillator (LO) as the receiver photodiode is operated under saturation for optimum mixing performance. The need for amplification can be reduced by improving fibre to chip coupling with a modeconverting optical waveguide integrated with the UTC-PD[5], or through monolithic integration with laser diodes[6].

Several improvements to the system are envisaged to increase the scope and effectiveness of the frequency domain spectrometer. In this case, two laser sources are used to optically pump both the receiver and emitter photodiode. Both are subject to a finite linewidth of $10 \mathrm{kHz}$ for laser 1 and 100 $\mathrm{kHz}$ for laser 2. In this case, the optical heterodyne signal generated in the photodiode is the convolution of the two free running laser linewidths. The current experimental arrangement is limited in linewidth of the RF signal to a resolution of $\sim 30$ $\mathrm{MHz}$ due to the jitter of the two lasers. By replacing the free running lasers with a coherent source such as an optical frequency comb and two high-quality tuneable optical filters such as an Optical Phase Lock Loop (OPLL), two coherent optical tones could be delivered to the photodiode with absolute spacing between them. Such an arrangement would allow for linewidths on the order of tens of $\mathrm{Hz}$ to be achieved[7], thereby increasing significantly the frequency resolution of the proposed spectrometer.

Additionally, the spectrometers frequency range of operation, which is currently limited by the bandwidth of the Electrical components used can also be overcome through integration of the photodiodes with a broadband antenna[8]. In this case the main challenge is the impedance matching of the device to the antenna, however, impedance matching circuits for UTC photodiodes have been already demonstrated for coplanar integrated devices[9].

\section{SUMMARY}

This system presents a proof of concept solution for noninvasive and continuous glucose concentration monitoring devices. Moreover, the system has a potential to be realized in a compact and portable form as in previously demonstrated monolithic integration of UTC-PDs with laser systems [6], which, have the potential to be used as wireless transceivers, due to the ability of the UTC photodiode to both generate and detect signals in a range of frequencies from microwave to $\mathrm{THz}$. Moreover, integration of the system would allow for enhanced sensitivity by removing power amplitude fluctuations from the unpackaged chip and improve polarization stability between the two lasers.

\section{REFERENCES}

[1] T. Ishibashi, T. Furuta, H. Fushimi, and H. Ito, "Photoresponse characteristics of uni-traveling-carrier photodiodes," 2001, vol. 4283, no., pp. 4211-4283.

[2] T. Nagatsuma et al., "Continuous-wave terahertz spectroscopy system based on photodiodes," PIERS Online, vol. 6, no. 4, pp. 390-394, 2010

[3] T. Torii, H. Chiba, T. Tanabe, and Y. Oyama, "Measurements of glucose concentration in aqueous solutions using reflected $\mathrm{THz}$ radiation for applications to a novel sub-THz radiation non-invasive blood sugar measurement method," Digit. Heal., vol. 3, p. 2055207617729534 , Jan. 2017.

[4] H. C. García et al., "Glucose sensing in saline solutions using Vband waveguides," in 2014 4th International Conference on Wireless Mobile Communication and Healthcare - Transforming Healthcare Through Innovations in Mobile and Wireless Technologies (MOBIHEALTH), 2014, pp. 320-323.

[5] E. Rouvalis, M. J. Fice, C. C. Renaud, and A. J. Seeds, "MillimeterWave Optoelectronic Mixers Based on Uni-Traveling Carrier Photodiodes," IEEE Trans. Microw. Theory Tech., vol. 60, no. 3 PART 2, pp. 686-691, Mar. 2012.

[6] G. Carpintero et al., "Microwave Photonic Integrated Circuits for Millimeter-Wave Wireless Communications," J. Light. Technol., vol. 32, no. 20, pp. 3495-3501, 2014.

[7] K. Balakier, L. Ponnampalam, M. J. Fice, C. C. Renaud, and A. J. Seeds, "Integrated Semiconductor Laser Optical Phase Lock Loops," IEEE J. Sel. Top. Quantum Electron., vol. 24, no. 1, pp. 112, 2018.

[8] C. C. Renaud, M. Natrella, C. Graham, J. Seddon, F. Van Dijk, and A. J. Seeds, "Antenna integrated THz uni-traveling carrier photodiodes," IEEE J. Sel. Top. Quantum Electron., vol. 24, no. 2, Mar. 2018

[9] H. Ito, S. Kodama, Y. Muramoto, T. Furuta, T. Nagatsuma, and T. Ishibashi, "High-speed and high-output InP-InGaAs unitravelingcarrier photodiodes," Sel. Top. Quantum Electron. IEEE J., vol. 10, no. 4, pp. 709-727, Jul. 2004. 